\title{
Condutas de enfermagem aplicadas ao paciente com infarto agudo do miocárdio no pré-hospitalar
}

\author{
Nursing conduct applied to pre-hospital patients with acute \\ myocardial infarction
}

\author{
Francisco Mayron Morais Soares' $\bullet$ Edson Juvenal da Silva Neto ${ }^{2}$ Kirley Kethellen Batista Mesquita $^{3}$ \\ Elison Denis Soriano Soares ${ }^{4} \bullet$ Davi Santos Magalhães ${ }^{5}$ Eryka Maria Rodrigues Pereira ${ }^{6}$ \\ Glaucirene Siebra Moura Ferreira ${ }^{7}$ Tatyane Oliveira Rebouças $^{8} \bullet$ Samylle Barbosa Veras Ferro $^{9}$
}

\begin{abstract}
RESUMO
O presente estudo teve como objetivo descrever condutas de enfermagem aplicadas ao paciente com infarto agudo do miocárdio no pré-hospitalar.Trata-se de uma revisão integrativa da literatura, com a metodologia proposta pelo Instituto Joanna Briggs (JBI), com busca nas bases de dados: Literatura Latino-Americana e do Caribe em Ciências da Saúde, National Library of Medicine, Scopus, ScientificElectronic Library Online e BDENF. Foram analisados 25 estudos, que atenderam os critérios de inclusão estabelecidos. Entre os diversos ganhos obtidos, destaca-se a atuação do enfermeiro na melhoria da prática clínica, a ampliação do conhecimento pelo enfermeiro especialista e uma melhoria do trabalho em equipe.As evidências demonstram a ampla possibilidade de atuação do enfermeiro na assistência de enfermagem aos pacientes com infarto do miocárdio. Palavras-chave: Infarto do Miocárdio; Serviços Médicos de Emergência; Cuidados de enfermagem.
\end{abstract}

\begin{abstract}
The present study aimed to describe the nursing practices applied to patients with acute myocardial infarction in the pre -hospital. It is an integrative literature review, with a methodology proposed by the Joanna Briggs Institute (JBI), with a search in the databases: Latin American and Caribbean Literature in Health Sciences, National Library of Medicine, Scopus, ScientificElectronic Library Online and BDENF. 25 studies were analyzed, which meet the included inclusion criteria. Among the various gains obtained, the participation of nursing professionals in improving clinical practice, the expansion of specialist nurse knowledge and an improvement in teamwork. As demonstrated, a wide possibility for nurses to act in nursing care for patients with myocardial infarction.
\end{abstract}

Keywords: Myocardial Infarction; Emergency Medical Services; Nursing Care.

\footnotetext{
I Enfermeiro. Mestre em Enfermagem pela Universidade da Lusofonia Afro-Brasileira - UNILAB. Professor da Graduação em Enfermagem da Faculdade UNINTA Itapipoca. Pesquisador GEPATE.

2 Enfermeiro. Especialista em Enfermagem Cardiovascular e Hemodinâmica. Serviço de Atendimento Móvel de Urgência - SAMUFOR

3 Enfermeira pela Universidade de Fortaleza. Mestranda em Enfermagem pelo Programa de Pós-graduação UFC. Membro GEPATE.

4 Graduando em Enfermagem pela Faculdade UNINTA Itapipoca. Membro GEPATE.

5 Graduando em Enfermagem pela Faculdade UNINTA Itapipoca. Bolsistas de Iniciação Científica - UNINTA. Membro GEPATE.

6 Enfermeira. Mestre em Saúde,Ambiente e Trabalho pela Universidade Federal da Bahia. Gestora Pedagógica do Curso de Enfermagem da Faculdade UNINTA Itapipoca.

7 Enfermeira. Doutoranda em Saúde Coletiva - UECE. Coordenadora do Curso de Enfermagem da Faculdade UNINTA Itapipoca.

8 Enfermeira do Centro de Hematologia e Hemoterapia do Ceará. Mestre em Ensino e Saúde - UECE.

9 Enfermeira. Especialista em Saúde da Mulher e Enfermagem Obstétrica.
} 


\section{INTRODUÇÃO}

A síndrome coronariana é uma doença aguda que afeta a musculatura estriada cardíaca, o diagnóstico e tratamento precoce são fatores essenciais para $\circ$ bom prognóstico do paciente, o sistema de saúde gasta anualmente milhões com pacientes vítimas dessa síndrome, ela pode ser dividida em angina instável e estável variando de acordo com a história clínica do paciente. Nos últimos anos houve uma queda no número de óbitos decorrente de investimento e novas terapias que prolongam a vida de pacientes sendo os cuidados de enfermagem fundamentais para a qualidade de vida '.

As doenças crônicas não transmissíveis foram responsáveis em 201 I por aproximadamente $74 \%$ do número total de óbitos no Brasil, estando as Doenças cardiovasculares (DCV) relacionadas a aproximadamente $1 / 3$ desse ${ }^{2}$.

As doenças de origem circulatória são um grave problema de saúde pública, dentre elas o Infarto Agudo do Miocárdio (IAM) é a mais conhecido sendo caraterizado por uma isquemia cardíaca e resulta em danos a curto e longo prazo para as vítimas, exigindo cuidados especializados durante o quadro agudo e cuidados contínuos por toda a vida ${ }^{3}$.

A isquemia miocárdica promove uma experiência singular para cada paciente, variando de uma relação entre fatores de risco, estado de saúde, ambiente e tratamento, o impacto dela na vida do paciente é enorme, exigindo dele uma mudança em vários aspectos da sua vida como alimentação e rotina familiar, cabe a equipe de saúde juntamente com a família e o próprio paciente formular estratégias de enfrentamento e adaptação para a nova realidade ${ }^{2}$.

A principal causa do IAM é a ruptura de uma placa aterosclerótica em uma artéria coronariana. Esse quadro, denominado arteriosclerose coronariana, é uma patologia com progressão lenta caracterizada pelo acúmulo lipídico que forma placas de ateroma e posteriormente obstrui o vaso sanguíneo com um processo envolvendo células de defesa e algumas substâncias que resulta em agregação plaquetária e formação trombo, esse bloqueio impede que os tecidos cardíacos recebam oxigênio e nutrientes resultando e sofrimento tecidual ${ }^{1,4}$.

O IAM atualmente é responsável por cerca de 100 mil óbitos anuais, a área tecidual acometida por isquemia quando não há intervenção, entra em um processo de morte celular perdendo permanentemente sua função e reduzindo o debito 5 .

O alto custo dos tratamentos de doenças cardiovasculares tem impactado negativamente o orçamento anual do Sistema Único de Saúde (SUS), os pacientes necessitam de cuidados complexos e profissionais qualificados e essa demanda tem gerado um imenso ônus para a saúde pública de todo o país 4 .

Para um cuidado efetivo pós infarto o enfermeiro deve realizar um planejamento de cuidado singular para cada paciente, atentando a seu contexto e suas peculiaridades, utilizando práticas assistenciais com embasamento científico que promovam a melhor e mais tranquila recuperação da saúde do paciente e todos os seus aspectos 6 .

Em razão da peculiaridade das DCV muitas famílias têm sua estrutura familiar alterada logo após o diagnósti$c o$, afetando negativamente o aspecto emocional da saúde do paciente, cabe a equipe de saúde oferecer suporte ao paciente e a qualquer membro de seu ciclo social que necessite, visando fortalecer os vínculos e estruturar o suporte social do paciente ${ }^{2}$.

Em emergência $\circ$ enfermeiro será $\circ$ primeiro profissional responsável a fazer a triagem desse paciente, cabendo a ele avaliação e determinação de prioridades bem como encaminhamento a área de tratamento ${ }^{7}$.

Segundo o protocolo Advanced Cardiac Life Support (ACLS), nos primeiros atendimentos ao paciente portador de IAM é importante que se mantenha e restabeleça a atividade cardíaca e respiratória. $O$ enfermeiro além de prestar atendimentos emergenciais atua também no monitoramento contínuo desse paciente, logo é de extrema relevância que o profissional de enfermagem tenha competência técnica para identificação de sinais e sintomas, interpretação de dados do eletrocardiograma (ECG), como também a prestação de cuidados de enfermagem ${ }^{8}$.

De acordo com estudo ${ }^{2}$, os profissionais da saúde em especial médicos e enfermeiros, atuam no cuidado ao portador de IAM por meio de ações instrumentais, assistenciais e de forma emocional, através de gestos de acolhimento e palavras de conforto. É relevante ressaltar que o apoio desses profissionais não se perpetua nas demais etapas de recuperação desse paciente, deixando de lado ações de promoção da saúde e do cuidado continuado.

Dessa forma é visto a importância da assistência de enfermagem ao paciente com Infarto Agudo do Miocárdio no atendimento pré-hospitalar, pois, além do enfermeiro atuar na identificação do IAM através de sinais e sintomas e interpretação de exames, o enfermeiro desenvolve competências também no processo de cuidados contínuos, é importante ressaltar que a sobrevida desses pacientes está diretamente relacionada a um atendimento de qualidade, logo a contribuição do cuidado clinico de enfermagem envolve conhecimentos científicos e habilidades técnicas ${ }^{9}$.

Para tanto, o presente estudo tem como por objetivo descrever condutas de enfermagem aplicadas ao paciente com infarto agudo do miocárdio no pré-hospitalar. 


\section{MÉTODO}

Trata-se de revisão integrativa, realizada pelo método do Instituto Joanna Briggs (JBI) ${ }^{10}$ que tem como foco a viabilidade, adequação, significância e eficácia das intervenções de saúde. Esse método pode ser utilizado para mapear os principais conceitos que sustentam uma área de pesquisa, bem como esclarecer as definições de trabalho e/ou limites conceituais de um tópico.

Para construção da pergunta de pesquisa, utilizou-se a estratégia PICO nos artigos quantitativos: $\mathrm{P}$-Pacientes com infarto agudo do miocárdio; I - transportados pelos serviços de emergência; $C$ - Assistência de enfermagem; O -Condição clínica do paciente.

Essa estratégia permitiu formular a seguinte questão norteadora: Quais as condutas de enfermagem ao paciente com infarto agudo do miocárdio no atendimento pré-hospitalar?

Dessa maneira, ao se estabelecer a pergunta, realizou-se uma busca inicial no portal de Bases de dados em Enfermagem BDENF para identificar os principais descritores e palavras-chaves utilizados nos estudos que abordassem a temática de interesse nesta revisão.

Para responder à questão de pesquisa, realizou-se a seleção dos descritores controlados e não controlados, relacionados com cada um dos componentes da estratégia PICO, utilizados de acordo com os descritores em Ciências da Saúde (DECS) e Medical Subject Headings (MeSH).

A pesquisa foi realizada de janeiro a março de 2019 , com restrições quanto ao espaço temporal dos últimos cinco anos, mas sem restrições ao tipo de apresentação ou de publicação com os descritores controlados: Infarto do Miocárdio; Serviços Médicos de Emergência; Cuidados de enfermagem; Myocardial Infarction; Emergency Medical Services; NursingCare.

Foram estabelecidos os critérios de inclusão e exclusão para a pesquisa, considerando-se os seguintes tipos de estudos: I) estudos realizados com pacientes com infarto; 2) estudos que abordassem a temática sobre infarto do miocárdio e assistência de enfermagem, 3) estudos com enfoque quantitativo e/ou qualitativo, que responderam à pergunta estabelecida, independentemente da área de conhecimento a que estivessem vinculados e 4) estudos publicados em português, inglês e espanhol. Foram excluídas as publicações de opiniões, consensos, retrações, editoriais e relatos de experiência.

Para identificar os estudos, foram utilizadas as seguintes bases de dados eletrônicas: Literatura Latino-Americana e do Caribe em Ciências da Saúde (LILACS), National Library of Medicine (PubMed), Scopus, ScientificElectronic Library Online (SciELO), Base de dados de Enfermagem (BDENF).

Foram selecionados na pesquisa com descritores individuais 12.298 artigos. Dentre estes, foram excluídos 4484 artigos por estarem publicados em duas ou mais bases de dados. Após o cruzamento dos descritores controlados, obteve-se uma amostra de 402 artigos. Após leitura exaustiva dos títulos e resumos dos artigos dos 402 estudos, 377 foram excluídos por não responderem à pergunta de pesquisa, e 25 foram selecionados para leitura na íntegra. Entre os 402 estudos analisados, 25 foram inclusos na pesquisa por responderem à pergunta e estarem em concordância com os critérios de inclusão estabelecidos.

A partir dessa seleção agruparam-se os artigos com base na semelhança dos dados em que foram organizados por três categorias: Fatores de risco e características clínicas do Infarto Agudo do Miocárdio, Intervenções de enfermagem durante $o$ transporte do paciente, Assistência de Enfermagem ao Paciente com Infarto Agudo do Miocárdio, possibilitando norteamento para as discussões que fundamentaram os resultados do estudo.

\section{RESULTADOS E DISCUSSÃO}

\section{Fatores de risco e características clínicas do Infarto Agudo do Miocárdio}

As doenças cardiovasculares são responsáveis pelo maior número de mortalidade em países desenvolvidos, onde a Síndrome Coronariana Aguda (SCA) corresponde a $42 \%$ desses óbitos, dentre tais síndromes o Infarto agudo do Miocárdio (IAM) se destaca como a principal causa de morte no Brasil.

O IAM é o resultado do desequilíbrio entre a oferta e a demanda de oxigênio pelo miocárdio". Esse desequilíbrio culmina em necrose celular da musculatura cardíaca. $O$ suprimento do miocárdio é realizado por um conjunto de vasos que tem origem na base da artéria aorta, esse vasos quando submetidos ao acúmulo anormal de substâncias lipídicas desenvolvem um processo inflamatório levando a formações de placas de ateroma, essa placa cria um bloqueio ou diminui a luz do vaso de modo a ocorrer a diminuição do suprimento sanguíneo para o miocárdio ${ }^{8}$.

A patologia é responsável por cerca de $6 \%$ a $10 \%$ das mortes no Brasil, mais de $50 \%$ dessas mortes ocorrem já em ambiente pré-hospitalar, onde $40 \%$ a $65 \%$ tem iminência de morte no início dos sintomas e aproximadamente $80 \%$ nas primeiras $24 \mathrm{~h}^{12-13}$. O tempo de atendimento é de fundamental importância para a melhora no prognóstico do paciente, pois a rápida restauração do fluxo sanguíneo limita a lesão do miocárdio reduzindo dessa forma 0 risco de morte e de complicações ${ }^{14}$.

A doença apresenta diversos fatores que podem levar ao seu surgimento, tais fatores são classificados entre fatores modificáveis e não modificáveis, os não modifi- 
cáveis são: etnia, idade, sexo, à genética e o histórico familiar. Os modificáveis são compostos por alimentação irregular, dieta rica em gordura, tabagismo, uso excessivo de bebidas alcóolicas, uso de drogas ilícitas, estresse e o sedentarismo ${ }^{15}$.

Em um estudo ${ }^{16}$ retrospectivo, com amostra de 412 pacientes, mostrou que a idade média dos participantes do estudo era de 68,4 apresentando idade mínima de 29 e máxima de 97 anos, a maioria eram do sexo masculino (68,9\%), em relação aos fatores de riscos o de maior prevalência foi a Hipertensão Arterial (68,7\%), seguido da dislipidemia (57,3\%), $41,5 \%$ dos pacientes eram fumantes ou já fumaram. Relacionado aos antecedentes cardíacos, $25,5 \%$ tinha algum tipo de antecedentes, sendo o IAM ० mais prevalente.

Nessa mesma perspectiva, uma pesquisa ${ }^{2}$ com o objetivo de identificar a presença de fatores de risco em pacientes com IAM, constatou que o fator de risco mais prevalente foi o sedentarismo $(91,7 \%)$, a hipertensão arterial sistêmica com $63,8 \%$, seguido de estresse (50\%), circunferência abdominal fora dos padrões de normalidade $(50 \%)$, tabagismo $(50 \%)$, sobrepeso $(35,5 \%)$ e obesidade (33,4\%). Relacionado ao histórico familiar, 43,7 \% dos participantes relataram ter um familiar com histórico de infarto agudo do miocárdio. Enquanto aos hábitos alimentares $\circ$ que mais destacou na pesquisa, foi o consumo diário de carne vermelha (56,3\%), e $37,5 \%$ dos participantes referiram fazer consumo de alimentos fritos na semana.

Tais fatores de riscos corroboram com vários outros estudos, que também objetivaram verificar suas ocorrências, identificou-se num estudo ${ }^{16}$ no estado do Piauí, que dentre os fatores de riscos mais ocorrentes, ser do sexo masculino apareceu com $56,2 \%$, idade superior a 60 anos $(59,8 \%)$, diabéticos $(29,6 \%)$, hipertensos (84,6\%), adeptos ao etilismo (6,7\%), tabagismo (35,4\%) e a dislipidemia (7,5\%), fator esse que em outros estudos surge em maior proporção.

A hipertensão arterial é considerada o fator mais importante para a ocorrência de doenças isquêmicas e para $o$ acidente vascular encefálico, assim também como o tabagismo; estudos mostram que 0 tabagismo entre pessoas de 60 anos de idade aumenta o dobro de chance de algum evento cardiovascular em comparação com os não tabagistas. Dessa forma, é visto que o controle dos fatores relacionados ao estilo de vida é fundamental para a prevenção dos eventos cardiovasculares ${ }^{17}$.

O Infarto Agudo do Miocárdio geralmente é desencadeado por esforço físico ou estresse emocional, sendo caracterizado clinicamente por sintomas como dor em aperto, sensação descrita como peso ou opressão localizada na região retroesternal ou precordial, que pode ser localizada também em região epigástrica, na mandí- bula, membro superior esquerdo, hemitórax direito, hipocôndrios e região dorsal. É uma dor intensa e pode ser acompanhada de palidez, sudorese, náuseas e vômitos ${ }^{18}$. A dor apresentada durante o IAM, é uma dor de origem isquêmica e é caracterizada pelo sinal de Levine (o paciente coloca sua mão espalmada sobre o centro do precórdio) ${ }^{19}$.

A morte tecidual cardíaca pode ser detectada pelo Eletrocardiograma (ECG), o tempo ideal de realização do ECG é de menos de 10 minutos depois da chegada na emergência e é o centro decisório para a confirmação na suspeita de um IAM, onde é possível a detecção de alterações em onda $T$, o infradesnivelamento e supradesnivelamento do seguimento ST e apresentação da onda $\mathrm{Q}$, todas as alterações refletem a ocorrência de infarto agudo do miocárdio ${ }^{18}$.

Outros marcadores importantes da presença de lesão miocárdica, são os marcadores biológicos como a troponina cardíaca e a creatinoquinase fração MB (CK $\mathrm{MB})$, tendo elevação três e seis horas após o início dos sintomas, apresentando sensibilidade diagnóstica de $50 \%$ três horas após os sintomas e de $80 \%$ seis horas após ${ }^{20}$.

\section{Intervenções de enfermagem durante o trans- porte do paciente}

O atendimento pré-hospitalar é definido como uma assistência prestada a pacientes que se encontram em quadros agudos, de natureza clínica, traumática e psiquiátrica, onde essa assistência acontece no ambiente extra-hospitalar, onde se não realizada de forma correta pode levar a sequelas e até mesmo a óbito do paciente atendido.

A assistência do enfermeiro no serviço pré-hospitalar não se restringe a apenas à assistência ao paciente com risco de morte, mas também a realização de atividades educativas como instrutor, contribuir com a revisão dos protocolos de atendimento, elaboração de material didático, atuação junto com a equipe multiprofissional, liderar e coordenar a equipe envolvida, além do uso de raciocínio clínico para tomadas de decisões dispondo de habilidades para executar as intervenções ${ }^{21}$.

De acordo com o protocolo preconizado pelo $(A C L S)^{8}$, o primeiro atendimento ao paciente portador de IAM objetiva o estabelecimento ou aperfeiçoamento da atividade respiratória e cardiovascular do sujeito. $A$ assistência de enfermagem a vítima de IAM se mostra essencial, pois possibilita um melhor prognóstico minimizando complicações futuras ${ }^{22}$. Logo, é relevante que o enfermeiro seja capaz de desenvolver uma abordagem inicial rápida, eficaz com fundamentação em conhecimentos teóricos/científicos ${ }^{8}$.

Souza, em seu estudo' de revisão bibliográfica sobre o papel do enfermeiro no atendimento de urgência 
e emergência, constatou que na literatura em contexto emergencial, a assistência de enfermagem ao portador de IAM relaciona-se a algumas condutas, como, proporcionar repouso e diminuição da ansiedade, preparar materiais para exames solicitados e para intubação orotraqueal, realizar eletrocardiograma (ECG) e monitorização cardíaca, agilizar exames laboratoriais solicitados, fornecimento de suporte ventilatório, punção de acesso venoso periférico e garantir a administração de medicamentos prescritos.

É preconizado como conduta no atendimento pré -hospitalar da Síndrome Coronariana Aguda (SCA), a realização da avaliação primária, mantendo o paciente tranquilo e com cabeceira elevada em $45^{\circ}$; oferecer $\mathrm{O} 2 \mathrm{com}$ fluxo de $4 \mathrm{l}$ por minuto se detectado desconforto ou oximetria de pulso $<94 \%$; realizar a avaliação secundária dando prioridade aos sinais vitais, a monitorização cardíaca, avaliação secundária e caracterização da dor (qualidade, localização e irradiação); realização de ECG de 12 derivações podendo considerar o ECG de $2^{\circ}$, segundo a opinião do Telecárdio; instalar acesso venoso periférico e realizar a abordagem medicamentosa com administração de AAS, clopidogel e dinitrato de isossorbida ${ }^{8}$.

\section{Assistência de Enfermagem ao Paciente com Infarto Agudo do Miocárdio}

As doenças cardiovasculares constituem-se como sendo uma das maiores causas de morbimortalidade no mundo, no Brasil, essa condição é considerada um problema de saúde pública, cerca de $30,6 \%$ dos óbitos no país são decorrentes de doenças circulatórias, sendo o Infarto Agudo do Miocárdio (IAM), responsável por 53,8 das mortes para cada 100 mil pessoas. Estudos estatísticos mostram que o número de óbitos por IAM aumentará de 17 milhões em 2008, para 25 milhões no ano de $2030^{23}$.

No nosso sistema de saúde as doenças cardiovasculares representam 19\% dos gastos públicos, sendo a terceira maior causa de internação via SUS (Sistema Único de Saúde). Dados do Departamento de Informática do Sistema Único de Saúde (DATASUS) mostra que em 2016 foram registrados 107.409 internações e 12.215 mortes por IAM ${ }^{24}$.

Segundo a American Heart Association, dentre as principais causas das doenças cardiovasculares estão os maus hábitos de saúde, que incluem: fumo/uso do tabaco, obesidade, sedentarismo, dietas ricas em gorduras e sódio, história familiar e genética de cardiopatias, altos índices de colesterol e outros lipídeos sanguíneos, hipertensão arterial sistêmica, diabetes mellitus e síndrome metabólica ${ }^{25}$.

O IAM apresenta alguns sintomas clínicos que facilitam sua identificação antes mesmo do seu diagnóstico, a dor retroesternal é uma dor típica da isquemia miocárdica acometendo o lado esquerdo do paciente e raramente o lado direito, restringindo toda a região a uma pequena área anginosa, a dor anginosa é relatada como uma sensação de aperto na região retroesternal com duração de 20 minutos ou várias horas na angina instável ${ }^{26}$.

Em situação de emergência o enfermeiro será o primeiro profissional responsável a fazer a triagem desse paciente, cabendo a ele avaliação e determinação de prioridades bem como encaminhamento a área de tratamento ${ }^{26}$.

Segundo o Advanced Cardiac Life Support (ACLS), nos primeiros atendimentos ao paciente portador de IAM é importante que se mantenha e restabeleça a atividade cardíaca e respiratória. O enfermeiro além de prestar atendimentos emergenciais atua também no monitoramento contínuo desse paciente, logo é de extrema relevância que o profissional de enfermagem tenha competência técnica para identificação de sinais e sintomas, interpretação de dados do eletrocardiograma ECG, como também a prestação de cuidados de enfermagem ${ }^{8}$.

De acordo com um estudo ${ }^{23}$, os profissionais da saúde em especial médicos e enfermeiros, atuam no cuidado ao portador de IAM por meio de ações instrumentais, assistenciais e de forma emocional, através de gestos de acolhimento e palavras de conforto. É relevante ressaltar que o apoio desses profissionais não se perpetua nas demais etapas de recuperação desse paciente, deixando de lado ações de promoção da saúde e do cuidado continuado.

Em um estudo ${ }^{8}$ sobre a atuação do enfermeiro no atendimento emergencial ao paciente com IAM, constatou segundo a opinião de enfermeiros, os principais pontos a serem realizados na sua assistência, dentre os cuidados foram citados a promoção de conforto ao paciente através de um leito em Unidade de Terapia Intensiva, propicia conforto e redução dos níveis de ansiedade, por conta do medo de morrer.

Ademais, a realização de exames, em especial o eletrocardiograma ECG, esse exame permite a análise de toda a atividade elétrica cardíaca, sendo possível a identificação de distúrbios isquêmicos; Suporte ventilatório, no IAM há oclusão coronariana o que diminui o aporte de oxigênio disponível para a atividade cardíaca; Garantia de acesso venoso e administração de medicamentos, como agentes trombolíticos e sulfato de morfina; Monitorização contínua, atentando-se para sinais e sintomas, depressão respiratória e ritmo cardíaco. Os profissionais também citaram a importância da qualificação profissional, como forma de prestar um cuidado qualificado aos usuários.

Dessa forma é visto a importância da assistência de enfermagem ao paciente com Infarto Agudo do Miocárdio, 
pois, além do enfermeiro atuar na identificação do IAM através de sinais e sintomas e interpretação de exames, o enfermeiro desenvolve competências também no processo de cuidados contínuos, é importante ressaltar que a sobrevida desses pacientes está diretamente relacionada a um atendimento de qualidade, logo a contribuição do cuidado clinico de enfermagem envolve conhecimentos científicos e habilidades técnicas (RIBEIRO, 20I6).

\section{CONCLUSÃO}

O grande número de pesquisas encontradas neste estudo demonstra que a assistência de enfermagem tem contribuído de forma significativa para a melhoria do quadro clínico de saúde, e os profissionais com expertises nas áreas de atendimento pré-hospitalar prestam uma melhor assistência a fim de garantir uma maior taxa de sobrevida dos pacientes.

Nesse processo, nas mais diversas áreas da saúde, e com interprofissionais diversos, os ganhos obtidos são variados, entre os quais se destacam a melhoria do quadro clínico, a melhoria das instabilidades cardíacas e, sobretudo, a melhor da taxa de sobrevida. As evidências demonstram a ampla possibilidade da atuação do enfermeiro na prática clínica. 


\section{REFERÊNCIAS}

I. Ribeiro KRA, Silva LP, Lima MLS. Conhecimento do infarto agudo do miocárdio: implicações para assistência de enfermagem. RevEnferm UFPI. 20 I6; 5(4):63-8.

2. Garcia, RP, et al.Apoio social frente á necessidade de cuidado após infarto do miocárdio. RevBrasEferm. 2015 jul-ago; 68(4):649-55.

3. Santos, BS., et al. Infarto agudo do miocárdio: abordagem com enfermeiros de uma unidade intensiva coronariana. Rev. Enferm. UFPE 2017; Oct-Dez; I2(I I): 5 I 53-55.

4. Mertins SM, Kolankiewiz ACB, Rosanelli CLSP, Loro MM, Poli G,Winkelman ER, et al. Prevalencia de factores de riesgoen pacientes con infarto agudo de miocárdio.AvEnferm [internet]. 2016; 34(I):30-8.

5. Gadéa SF, Rodrigues M, Silva GC, Junior EA. Reabilitação cardíaca após infarto agudo do miocárdio (IAM): uma revisão sistemática. Revista Ciência 20I7; I (5).

6. Vargas, RA., et al. Qualidade de vida de pacientes pós-infarto do miocárdio: revisão integrativa da literatura. RevEnfermUFPEOnLine, 2019; 7(I I): p.2803-09.

7. Quaresma A dos S, Xavier DM, Vaz MRC-. O papel do enfermeiro na classificação de risco nos serviços de urgência e emergência. Revista Enfermagem Atual [Internet]. 8abr.2019 [citado 4maio2020];87(Edição Esp). Available from: https://revistaenfermagematual.com/index.php/revista/article/view/I5I.

8. American Heart Association, Inc. Circulation of the journal American Heart Association. Atualização das Diretrizes de RCP e ACE, Dallas,.

9. Ribeiro, K. R. A, Silva, L. P, Lima, M. L. Conhecimento do infarto agudo do miocárdio: Implicações para assistência de enfermagem. Revista de Enfermagem da UFPE. 2016; 5(4):63-8

10. Joanna Briggs Institute. The Joanna Briggs Institutebestpracticeinformationsheet: Music as anintervention in hospitals. Nursing\&healthsciences, 201 I; I3(I), 99-102.

II. Carvalho, Ana Teresa Glaser et al. Correlação entre Atividade Física e Variáveis Clínicas de Pacientes com Infarto Agudo do Miocárdio. Int J CardiovascSci, 2018; 3 I ( I):22-25.

12. Santos, C.A.Terapia trombolítica no IAM: uma revisão da literatura. Revista Recien-Revista Científica de Enfermagem, 2017; 7(20), 22-30.

13. Figueiredo, Ana Elizabeth et al. Determinação do tempo de apresentação a emergência de pacientes com infarto agu- do do miocárdio. Revista de Enfermagem da UFSM, 2013 3(1):93-101.

14. Santos, Bruno da Silva et al. Infarto agudo do miocárdio: abordagem com enfermeiros de uma unidade intensiva coronariana. Rev. Enferm. UFPE, 2017; I2(II): 5I53-5I55. 15. Sobrinho, José Renaldo Prata et al. Infarto agudo do miocárdio: uma revisão bibliográfica. ScireSalutis, 2015; 5(I):6-135.

16. Soares, Sónia Oliveira de Matos; ferreira, Paulo Alexandre Carvalho. A pessoa com enfarte agudo do miocárdio no serviço de urgência: fatores que influenciam o tempo de atendimento. Revista de Enfermagem Referência, 2017;(I5):3 I-42.

17. Paula, et al. Avaliação do risco cardiovascular em hipertensos. Revista Latino-Americana de Enfermagem, 2013; 2 I (3):820-8273.

18. Siervuli, Marcos Tadeu Ferreira et al. Infarto do miocárdio: alterações morfológicas e breve abordagem da influência do exercício físico. RevBrasCardiol, 20 I4; 27(5):349-55.

19. Passinho, R. S, SIPOLATTI,W. G. R, et al. Sinais, sintomas e complicações do infarto agudo do miocárdio. Rev enferm UFPE, 20 I8; I2(I): 247-64.

20. Da Costa, et al. Perfil demográfico de pacientes com infarto agudo do miocárdio no brasil: revisão integrativa. Sanare -Revista de Políticas Públicas, 20 I8; 17(2).

2 I. Souza, P. R.; chagas, H. O. The role of nurses in urgency and emergency care: A review of literature. ScientificElectronicArchives, 2018; I I (4):99- 105.

22. Friguini, et al. A sistematização da assistência de enfermagem e atuação do enfermeiro ao paciente infartado. Salus J Health Sci. 2016; 2(3): I-I3.

23. Garcia, R. P, budó, M. L. D, simon, B. S, et al.Vivências da família após infarto agudo do miocárdio. Rev Gaúcha enferm. 20। 3; 34(3): I7I- I78.

24.Vargas, Rodrigo Abreu de et al. Qualidade de vida de pacientes pós-infarto do miocárdio: revisão integrativa da literatura. Rev EnfermUFPE, 20 I7; 07(I I):2803-2809.

25. Passinho, R. S, sipolatti,W. G. R, et al. Sinais, sintomas e complicações do infarto agudo do miocárdio. Rev enferm UFPE, 2018; I2(I): 247-64.

26. Medeiros, T. C. F, andrade, P.C. N.S, et al. Mortalidade por infarto agudo do miocárdio. Rev enferm UFPE, 20।8; I2(2): 565-72.

Recebido: 2020-03-19 Aceito: $2020-05-20$ 